\title{
Neuroinflammation in neurodegenerative disorders: the roles of microglia and astrocytes
}

\author{
Hyuk Sung Kwon ${ }^{1}$ and Seong-Ho Koh ${ }^{1,2^{*}}$ (D)
}

\begin{abstract}
Neuroinflammation is associated with neurodegenerative diseases, such as Alzheimer's disease, Parkinson's disease, and amyotrophic lateral sclerosis. Microglia and astrocytes are key regulators of inflammatory responses in the central nervous system. The activation of microglia and astrocytes is heterogeneous and traditionally categorized as neurotoxic (M1-phenotype microglia and A1-phenotype astrocytes) or neuroprotective (M2-phenotype microglia and A2phenotype astrocytes). However, this dichotomized classification may not reflect the various phenotypes of microglia and astrocytes. The relationship between these activated glial cells is also very complicated, and the phenotypic distribution can change, based on the progression of neurodegenerative diseases. A better understanding of the roles of microglia and astrocytes in neurodegenerative diseases is essential for developing effective therapies. In this review, we discuss the roles of inflammatory response in neurodegenerative diseases, focusing on the contributions of microglia and astrocytes and their relationship. In addition, we discuss biomarkers to measure neuroinflammation and studies on therapeutic drugs that can modulate neuroinflammation.
\end{abstract}

Keywords: Neuroinflammation, Neurodegenerative diseases, Microglia, Astrocytes

\section{Background}

With the increase in life expectancy, the global socioeconomic impact of neurodegenerative diseases, including Alzheimer's disease (AD), Parkinson's disease (PD), and amyotrophic lateral sclerosis (ALS), is increasing considerably [1]. However, the pathological mechanisms underlying neurodegenerative diseases are not fully understood. Several factors including genetic, environmental, and endogenous factors are involved. Abnormal protein dynamics, oxidative stress with reactive oxygen species, mitochondrial dysfunction, DNA damage, dysfunction of neurotrophins, and neuroinflammatory processes are considered to be common pathophysiological

\footnotetext{
* Correspondence: ksh213@hanyang.ac.kr

'Department of Neurology, Hanyang University College of Medicine, Seoul, Republic of Korea

${ }^{2}$ Department of Translational Medicine, Hanyang University Graduate School of Biomedical Science \& Engineering, Seoul, Republic of Korea
}

mechanisms [2]. Neuroinflammation is a defense mechanism that initially protects the brain by removing or inhibiting diverse pathogens [3]. This inflammatory response can have beneficial effects by promoting tissue repair and removing cellular debris. Sustained inflammatory responses, however, are detrimental, and they inhibit regeneration $[4,5]$. Inflammatory stimulation can persist due to endogenous (e.g., genetic mutation and protein aggregation) or environmental (e.g., infection, trauma, and drugs) factors [6, 7]. The persistent inflammatory responses involve microglia and astrocytes and can lead to neurodegenerative diseases [4].

Two categories of cells populate the central nervous system: neurons and glial cells [8]. Glial cells do not produce electrical impulses, and they were considered as supporting cells for neurons. It has been revealed that glial cells are superior to neurons in cellular diversity and function [9]. Glial cells, including astrocytes, 
oligodendrocytes, and microglia, can regulate neuronal activity $[8,10]$. Microglia and astrocytes serve diverse functions including innate immune responses in the brain. Traditionally, both can be classified into two opposing phenotypes: neurotoxic and neuroprotective. Microglia are divided into the M1 (classical activation) and M2 (alternative activation) phenotypes based on their activation status $[6,11]$. Similar to the microglia, astrocytes can produce pro-inflammatory or immunoregulatory mediators according to the phenotype of the polarization status [7]. However, microglia and astrocytes are considered to have multiple reactive phenotypes related to the type and stage of neurodegenerative diseases and the regional location [12-14]. Furthermore, the changes in phenotypes of microglia and astrocytes, their loss of neuroprotective functions, and their gain of neurotoxic functions are complicated and may differ with the stage and severity of neurodegenerative diseases. Therefore, the simple dichotomized classification cannot reflect the various phenotypes of microglia and astrocytes [12]. For these reasons, the use of the M1/M2 and A1/A2 nomenclature was limited in this manuscript, appearing only directly from the references where they were used. However, they should be considered as being on a spectrum, rather than being two distinct populations. This complexity could be the reason why trials of anti-inflammatory drugs have, to date, failed to show significant therapeutic effects. Here, we review the roles of inflammatory responses in neurodegenerative diseases, such as $\mathrm{AD}$, $\mathrm{PD}$, and ALS, focusing on the roles of microglia and astrocytes and their relationships. Recommendations for the success of clinical trials are also made. In addition, biomarkers to measure neuroinflammation and studies on drugs that can modulate neuroinflammation are also discussed.

\section{Microglia}

Microglia are ubiquitously distributed in the brain and are the principal innate immune cells and the first responders to pathological insults $[15,16]$. The proportion of microglia ranges $5-12 \%$ of the total cell population in the mouse brain depending on the location, and they have diverse morphologies: compact round, longitudinally branched, and radially branched [17]. They are involved in homeostasis and host defense mechanisms by participating in three essential functions [18]. The first function is detecting changes in their environment using their sensomes, which are encoded by various genes [19]. The second is the physiological housekeeping function, which includes migrating to injured sites, remodeling synapses, and maintaining myelin homeostasis $[18,20]$. The third is protecting against injurious stimuli, including pathogen-associated molecular patterns (PAMPs) and damage-associated molecular patterns (DAMPs). Cellular receptors such as toll-like receptors (TLRs), nuclear oligomerization domain-like receptors, and viral receptors are expressed on microglia, and can recognize PAMPs and DAMPs [6, 7]. In response to such stimuli, microglia produce proinflammatory cytokines, such as tumor necrosis factor (TNF)- $\alpha$, interleukin (IL)-1 $\beta$, IL-16 and chemokines, including the $\mathrm{C}-\mathrm{C}$ motif chemokine ligand 2 (CCL2) and IL18 , to recruit additional cells and remove pathological agents $[6,18]$. However, although neuroinflammation is a neuroprotective mechanism, sustained neuroinflammation can induce neurotoxicity and is related to neurodegeneration [18]. In addition, microglia priming with aging and chronic stress shows a dystrophic morphology and an exaggerated inflammatory response [21].

Microglial activation can be assessed by imaging and fluid biomarkers. ${ }^{11} \mathrm{C}$-(R)PK11195 positron emission tomography (PET) can be used to quantify microglial activation via the binding capacity of ${ }^{11} \mathrm{C}$-(R)PK11195 to the translocator protein that is overexpressed in activated microglia $[22,23]$. The soluble triggering receptor expressed on myeloid cells 2 (sTREM2), which is a cleavage product of TREM2 expressed on the cell surface of microglia [24, 25], is a fluid biomarker of microglial activation. Recent studies have shown that the cerebrospinal fluid (CSF) level of sTREM2 is correlated with plasma sTREM2 level, suggesting that the CSF sTREM2 is a potential biomarker for microglial activation $[25,26]$.

Microglia in the central nervous system (CNS) can be pro-inflammatory or neuroprotective, depending on their activation status. Pro-inflammatory cytokines are debris from pathogens or damaged cells, and they activate the resting microglia to express pro-inflammatory factors such as IL-1 $\beta$, TNF- $\alpha$, IL- 6 , nitric oxide (NO), and proteases, which have detrimental effects in neurodegenerative diseases (Fig. 1, Fig. 2) [6, 13]. In contrast, IL-4, IL-10, IL-13, and transforming growth factor- $\beta$ (TGF- $\beta$ ) activate neuroprotective microglia, which leads to the release of diverse factors including FIZZ1, Chitinase-3-Like-3 (Chi313), Arginase 1, Ym1, CD206, insulin-like growth factor 1 (IGF-1), and Frizzled class receptor 1 (Fzd1) (Fig. 2) [6, 13, 27, 28]. These factors from microglia may be associated with neuroprotection and tissue healing (Fig. 3). For example, IL-4 is known to suppress the release of pro-inflammatory cytokines, i.e., IL-6, TNF- $\alpha$, and NO $[29,30]$.

Switching between these two phenotypes may affect remyelination, which is associated with aging $[7,31]$. Obesity, insulin resistance, and type 2 diabetes are known to impact the transition of microglia from the neuroprotective phenotype to the neurotoxic phenotype (Fig. 3) [28, 32, 33]. Several candidates and factors have been identified to potentiate the neuroprotective polarity: fasudil (Rho kinase inhibitor), Jumonji domaincontaining 3 (Jmjd3, H3K27me3 demethylase), minocycline, Copaxone (glatiramer acetate), dimethyl fumarate (Tecfidera), cromolyn, CHF 5074, fingolimod, masitinib, glycogen synthase kinase-3 inhibitor, histone deacetylase 


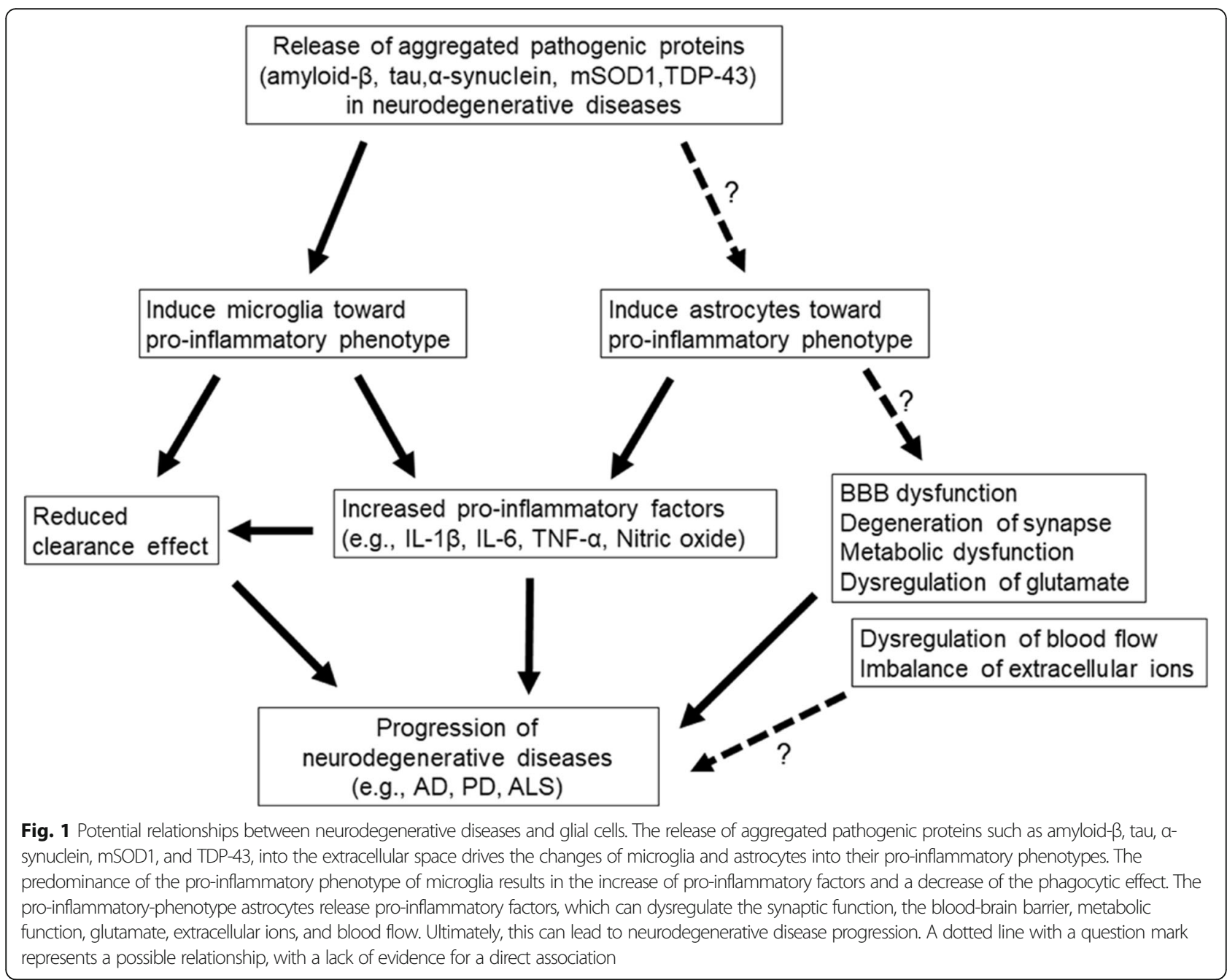

inhibitor, peroxisome proliferator-activated receptor, adenosine monophosphate-activated protein kinase, and Janus kinase/signal transducers and activators of transcription (JAK/STAT) inhibitors (Fig. 3) [34-39]. The proportion of each phenotype can differ depending on the stage of neurodegenerative diseases [28]. Treatment targeting the phenotype balance may have different effects, depending on the time window [28]. Therefore, balancing and switching between the phenotypes of microglia at specific times and in specific patients may be important for modulating the progression of neurodegenerative diseases. The drugs that modulate microglial activation are more likely to exhibit protective effects in a clinical trial that 1) employ participants with more pro-inflammatory than neuroprotective microglial phenotypes, 2) enroll participants who are likely to show progression within a few years, as it is hard to follow patients for longer duration of years in clinical trials, and 3) have confirmed the pathology of the disease, such as amyloidopathy or tauopathy; without a pathological insult, the glial cells may not change. Further studies are warranted to investigate the appropriate time window and patients to demonstrate the clinical benefits of treatments targeting the microglial state.

\section{Astrocytes}

Astrocytes are the most common glial cells in the brain [40]. Although they were initially considered to only have passive functions, recent studies have discovered that astrocytes play active and essential roles in brain homeostasis [41]. They regulate blood flow, maintain the blood-brain barrier (BBB), provide energy metabolites to neurons, modulate synaptic activity, control neurotrophin secretion, remove dead cells, as well as regulating the extracellular balance of ions, fluid and transmitters, and scar formation [40-42]. Currently, glial fibrillary acidic protein (GFAP), S100B, YKL040, and D-serine are assessed as CSF biomarkers and GFAP and S100B as blood biomarkers [43]. For imaging biomarkers, magnetic resonance spectroscopy, ${ }^{11} \mathrm{C}$-deuterium- $L$ - 


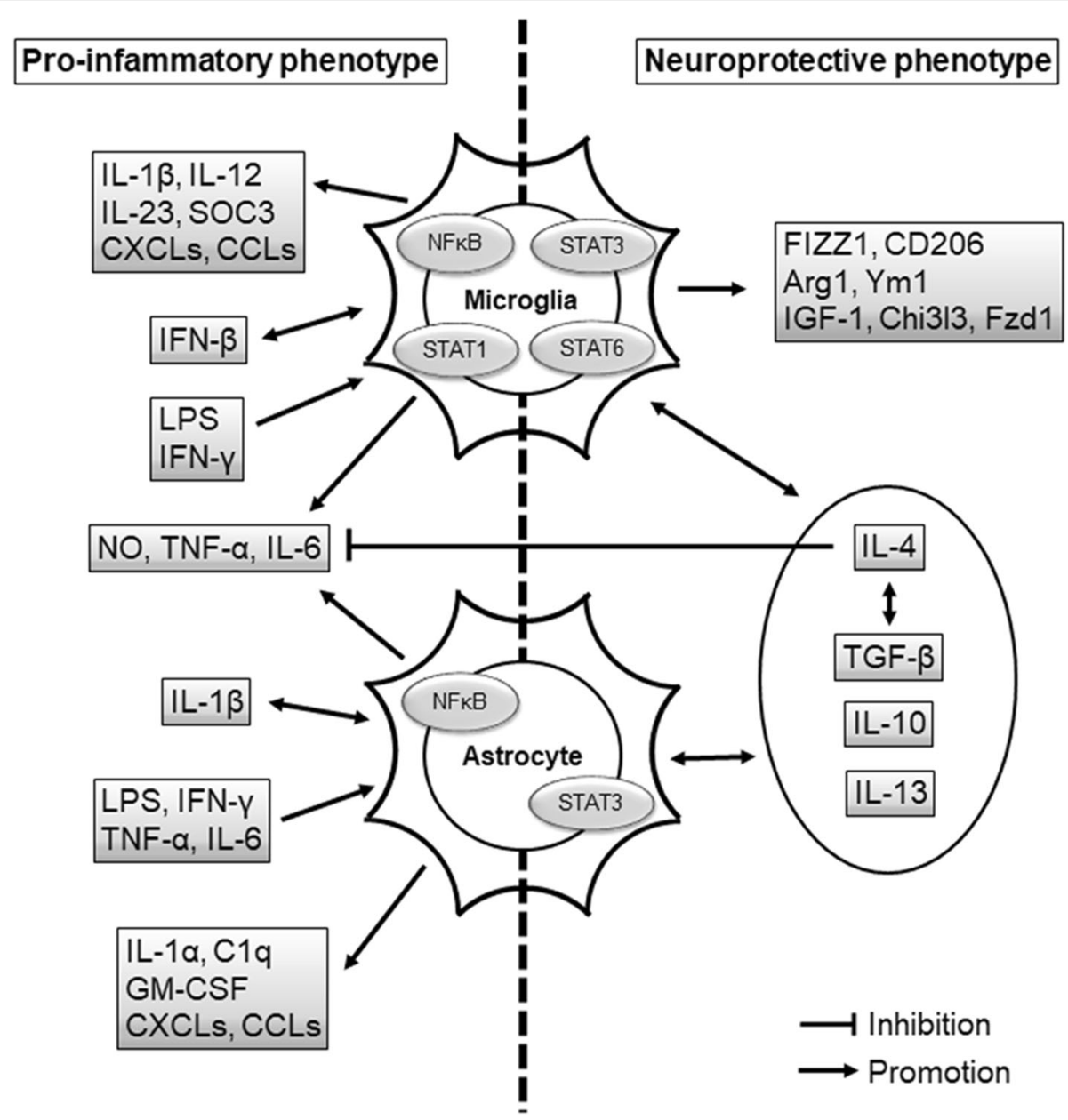

Fig. 2 Proposed signals associated with microglia and astrocytes. The pro-inflammatory microglia are activated by IFNs and LPS via the activation of NFKB and STAT1, and then release IL-1 $\beta, I L-12, I L-23, S O C 3, C X C L S, C C L s, N O$, TNF- $a$, and IL-6. The neuroprotective microglia are promoted by IL-4, IL-13, IL-10, and TGF- $\beta$ via the activation of STAT3 and STAT6. The M2 microglia enhance the neurotrophic factor (IGF-1), FIZZ1, CD206, Arg1, Ym1, Chi3|3, Fzd1, IL-13, IL-10, IL-4, and TGF- $\beta$. The activation of NFKB induces pro-inflammatory astrocytes. The pro-inflammatory astrocytes are affected by IL-1 $\beta$, IFN- $\gamma$, LPS, TNF- $a$, and IL-6, and they produce IL-1a, C1q, GM-CSF, CXCLs, CCLS, TNF- $a$, IL-6, and NO. The activation of STAT3 induces neuroprotective astrocytes. The neuroprotective astrocytes interact with anti-inflammatory cytokines such as IL-13, IL-10, TGF- $\beta$, and IL-4; IL-4 and TGF- $\beta$ coordinate to promote protective effects, and IL-4 suppresses TNF-a, IL-6, and NO. CCL: C-C-motif chemokine ligand; CXCL: C-X-C motif chemokine ligand; GM-CSF: granulocyte-macrophage colony-stimulating factor; IFN-ү: interferon ү; IL: interleukin; LPS: lipopolysaccharide; NFkB: nuclear factor KB; NO: nitric oxide; STAT: signal transducers and activators of transcription; TNFa: tumor necrosis factor a

deprenyl $\left({ }^{11} \mathrm{C}\right.$-DED) PET, and ${ }^{11} \mathrm{C}$-BU PET are used to assess the astrocyte reactivity [43]. Changes in molecular expression and morphology of astrocytes measured by GFAP can indicate the severity of reactive astrogliosis, which is a hallmark of CNS pathology [42]. Defects of astrocytes during the early phase of injury including spinal cord injury (SCI) and experimental autoimmune encephalomyelitis (EAE), are consistently correlated with exacerbated clinical outcomes, neuroinflammation, BBB alteration, and neuronal death [40], while on the other side, a study in a chronic experimental EAE mouse model has shown that during chronic CNS inflammation, astrocytes produce lactosylceramide (LacCer), which promotes inflammation and neurodegeneration
[44]. These results indicate that the effect of astrogliosis can be beneficial or detrimental, depending on the time, specific disease, and different stimuli from the microenvironment, such as microglia.

Astrocytes may have multiple simultaneous reactive profiles, but with a continuous spectrum. Therefore, the heterogeneity of reactive astrocytes should be investigated further [13]. Similar to microglia, astrocytes have pro-inflammatory and immunoregulatory (neuroprotective) subpopulations. The pro-inflammatory reactive astrocytes upregulate several genes (e.g., complement cascade genes) and induce proinflammatory factors (e.g., IL-1 $\beta$, TNF- $\alpha$, and NO), which are known to have harmful functions (Fig. 1) [6, 


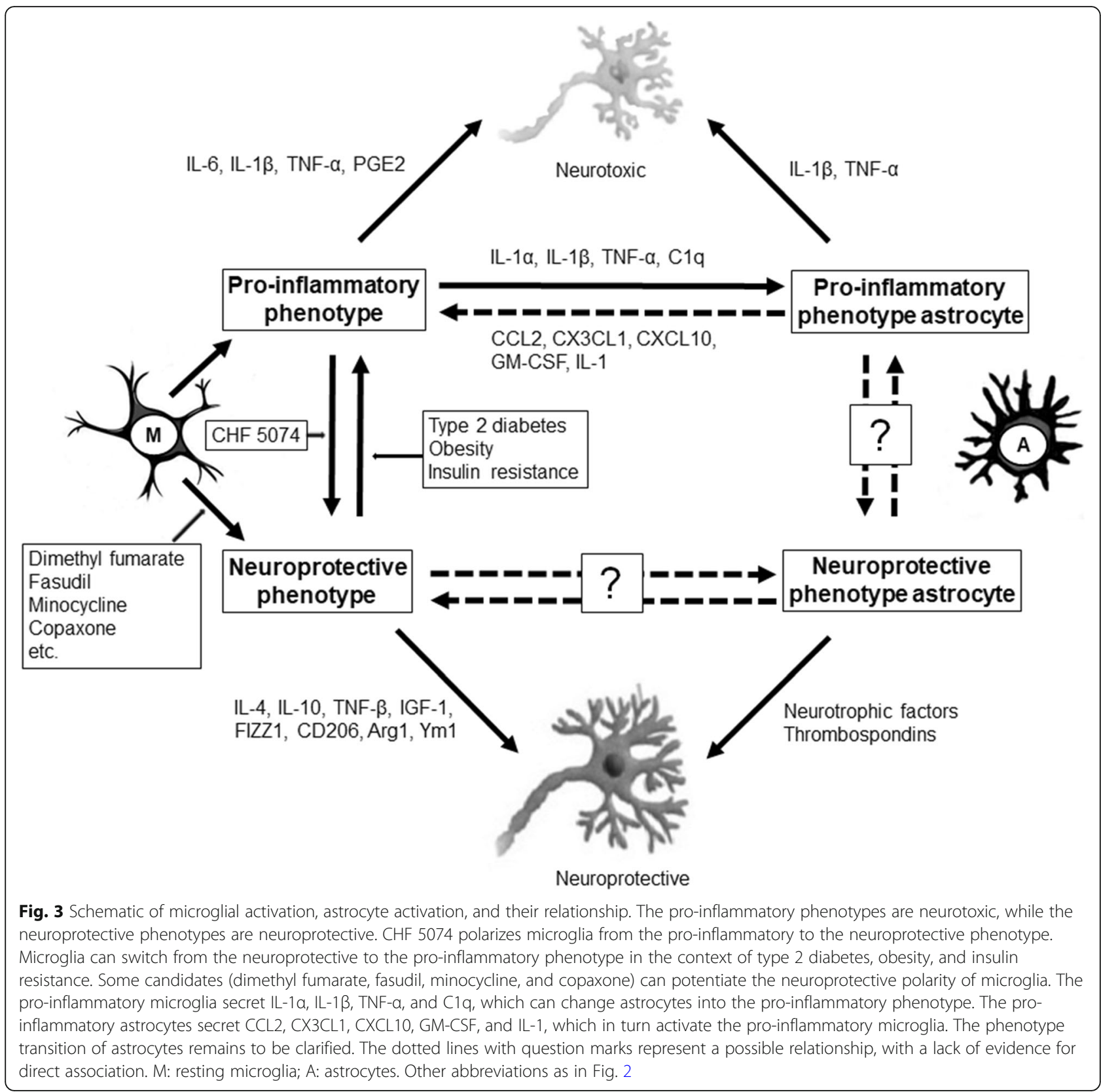

13]. In comparison, the neuroprotective reactive astrocytes upregulate many neurotrophic factors and thrombospondins (Fig. 3) [13]. The anti-inflammatory cytokines, such as IL-4, IL-13, and IL-10, may induce neuroprotective activation of astrocytes, and these alternatively activated astrocytes may release IL-4, IL10, and TGF- $\beta$ (Fig. 2) [41].

Inflammatory mediators secreted by pro-inflammatory microglia, such as IL- $1 \alpha$, IL- $1 \beta$, TNF- $\alpha$, and C1q, may activate pro-inflammatory astrocytes and induce a secondary inflammatory response (Fig. 3) [45, 46]. Detrimental astrocytic signaling pathways can be induced by several other cytokines, sphingolipids (sphingosine 1- phosphate and LacCer), and neurotrophins [40]. Astrocytes upregulate the transmembrane receptors for IL-17 and tropomyosin receptor kinase B (TrkB) during neuroinflammation. The binding of IL-17 to its receptors may result in the recruitment of nuclear factor $\kappa B(N F \kappa B)$ activator 1 (Act1) and the production of pro-inflammatory cytokines [47]. Conditional mice lacking TrkB can be protected from EAE-induced neurodegeneration, while stimulation of TrkB by the agonist brain-derived neurotrophic factor (BDNF) has detrimental effects on neurons [48].

In contrast, astrocytes that respond to certain pathways are protective, since inhibition of the mediators of 
these protective pathways worsens neuroinflammation and neuronal cell death. The first protective pathway is mediated by glycoprotein gp130; it is related to the SHP2/Ras/ERK activation, and limits neuroinflammation [49]. A lack of gp130, a signal transducer for the IL-6 cytokine family, worsens the CNS injury of Toxoplasma encephalitis and EAE in mice $[49,50]$. The second protective pathway is mediated by TGF $\beta$, which has important immunosuppressive properties. The astrocytic TGF$\beta$ signaling may mediate the inhibition of NFKB signaling and reduce neuroinflammation after a stroke or Toxoplasma infection $[51,52]$. The third protective pathway is mediated by interferon (IFN) $-\gamma$ signaling. Although IFN- $\gamma$ is a pro-inflammatory cytokine, the inhibition of its signaling in astrocytes worsens the mortality and leukocyte infiltration during the late stage of EAE in mice [53]. Finally, the estrogen receptor (ER) $\alpha$ signaling pathway in astrocytes has demonstrated antiinflammatory and neuroprotective effects in various neurological disease models [54].

The transcription factor signal transducer and activator of transcription 3 (STAT3) is expressed in astrocytes and phosphorylated after injury. Ablation of STAT3 in astrocytes aggravates the infiltration of inflammatory cells, neuronal loss, and demyelination after SCI in mice [55]. BDNF secreted by activated astrocytes can enhance STAT3 activation [56]. In another in vivo and in vitro study involving an SCI mouse model, the knock-out of STAT3 attenuated astrogliosis and disrupted scar formation, which were associated with the worsening of inflammation and increased lesion volume [57]. Therefore, STAT3 seems to be critically involved in reactive astrogliosis, and is associated with a neuroprotective effect.

The JAK-STAT3 pathway may mediate the neuroprotective functions of reactive astrocytes. However, the molecular basis for the induction of the neuroprotective reactive astrocytes is unclear [58]. In addition, there could be more states of polarization than just proinflammatory or neuroprotective [13]. Therefore, the heterogeneity and molecular basis of reactive astrocytes should be investigated further.

\section{$A D$}

$\mathrm{AD}$ is the most common form of dementia and is pathologically characterized by extracellular accumulation of amyloid-beta $(A \beta)$-containing plaques and development of intracellular neurofibrillary tangles composed of hyperphosphorylated tau protein $[16,59]$. In addition, neuroinflammation contributes to the pathogenesis of $\mathrm{AD}$ [16], as inflammatory responses have been repeatedly demonstrated in AD. For example, researchers have found higher TNF- $\alpha$ (pro-inflammatory cytokine) and lower TNF- $\beta$ (anti-inflammatory cytokine) levels in the CSF of mild cognitive impairment patients who progressed to $\mathrm{AD}$, compared with the controls who did not progress to $A D$ [60]. Some cytokines including IL$1 \beta$, IL-6, and TNF- $\alpha$ have slowly increased levels from the early stage of the disease, while the levels of other cytokines including IL-18, MCP-1, and IP-10 can peak at a certain stage of the disease [61]. Although current publications have inconsistent results, it has been clear that neuroinflammation occurs early in $\mathrm{AD}$, and may trigger the progression of this disease.

Morphological changes of microglia and astrocytes surrounding the senile plaques are also indicative of the neuroinflammatory response [6]. Both microglia and astrocytes interact with $A \beta$. Dysfunctions of microglia and astrocytic metabolism can result in the accumulation of $\mathrm{A} \beta[18,62] . A \beta$ in turn activates microglia and astrocytes through TLRs to release neuroinflammatory mediators that promote neurodegeneration $[4,6]$.

Microglia can be neuroprotective by degrading and removing $A \beta$ and tau $[63,64]$. However, the persistent interaction between $A \beta$ and $A \beta$-induced pro-inflammatory cytokines overwhelms the clearance ability of microglia [18]. Increases in the size and number of $A \beta$ plaques during the late-onset form of AD may reflect the decreased clearance ability of microglia [65]. Microglia that surround the $A \beta$ plaques are generally of the neuroprotective phenotype, labeled as $\mathrm{Ym} 1$, at the beginning of $A \beta$ pathology; but they later switch to the neurotoxic (pro-inflammatory) phenotype during the advanced stage of the disease $[28,66]$. The pro-inflammatory cytokines decrease the phagocytic activity of microglia, and they are also likely to transform microglia into the pro-inflammatory phenotypes. In addition, the pro-inflammatory microglia increase the phosphorylation of tau and exacerbate tau pathology [67]. This indicates that microglia in AD are involved in the $A \beta$ and tau pathologies. In a recent study, microglial activation, as measured by ${ }^{11} \mathrm{C}$-(R)PK11195, decreased longitudinally in patients with mild cognitive impairment and increased longitudinally in AD patients [68], suggesting that two peaks of microglial activation may be present in AD. Although the ${ }^{11} \mathrm{C}$-(R)PK11195 cannot differentiate between the phenotypes of microglial activation, the first and last peaks may be neuroprotective and proinflammatory, respectively, as microglia are known to change from the neuroprotective to the pro-inflammatory activation phenotypes during aging $[68,69]$. In addition, the microglial activation is significantly correlated with amyloid deposition (measured by ${ }^{11} \mathrm{C}$-PIB PET) [68], but not significantly correlated with tau accumulation (measured by $\left.{ }^{18} \mathrm{~F}-\mathrm{AV}-1451 \mathrm{PET}\right)$ in $\mathrm{AD}$ [23]. This difference can be explained by the stage of the disease and the regional location in the brain. CSF sTREM2, a marker of microglial activation, is also increased in $\mathrm{AD}$ patients compared with the healthy controls [25]. However, several studies on sTREM2 have contradictory findings and the 
discriminative power of sTREM2 for AD is low. Further studies are required to establish the utility of sTREM2 in clinical practice [70].

Pro-inflammatory reactive astrocyte phenotypes have been linked to synaptic degeneration and glutamate dysregulation [13, 41]. Knockout of astrocytic glutamate transporters EAAT1 (glutamate/aspartate transporter, GLAST) and EAAT2 (glutamate transporter-1, GLT-1) caused excitotoxicity and synaptic hyperexcitability in an AD model [71-73]. In the AD mouse model, calcineurin $\left(\mathrm{Ca}^{2+} /\right.$ calmodulin-dependent phosphatase $)$, a nuclear factor of the activated T-4 cell signaling pathway, has been found to link between astrocyte activation and hyperexcitability during $\mathrm{AD}$ [72]. The astrocytes alter their function and morphology during $\mathrm{AD}$, and may have different functions as AD progresses [43].

Most clinical trials for anti-inflammatory drugs in $\mathrm{AD}$ patients, including aspirin, prednisone, naproxen, diclofenac, indomethacin, and celecoxib, have failed to show definite improvements, even revealing some detrimental effects [74-79], although some have shown modest beneficial results. A subgroup analysis showed that mild-to-moderate $\mathrm{AD}$ patients who were $A P O E \varepsilon 4$ carriers benefitted from ibuprofen over the 12-month trial duration [80]. Another study revealed protective effects of indomethacin in mild-to-moderate $\mathrm{AD}$ patients over 6 months, but the patient dropout rate was high, which weakened the significance of the study [81]. The failure of the clinical trials may be explained by the possible impact of the anti-inflammatory drugs on the protective effects of glial cells, the selection of participants, and the shortterm follow-up period. For example, the Alzheimer's Disease Anti-inflammatory Prevention Trial (ADAPT) enrolled old cognitively unimpaired individuals and evaluated the effects of naproxen and celecoxib on cognitive function [79]. Although the participants were family members of $\mathrm{AD}$ patients, we did not know if they had $\mathrm{AD}$, and cognitive impairment may not have started in them; hence, microglia and astrocytes may have been more neuroprotective than proinflammatory. The COX-inhibiting anti-inflammatory drugs may reduce microglial activation [82]. Furthermore, 3 years of follow-up may not be long enough for revealing a difference, as cognitive decline may not start in participants within 3 years. Minocycline, which can cross the BBB and inhibit pro-inflammatory microglia [83], showed beneficial effects on memory impairment caused by $A \beta$ and reversed the increase in various inflammatory cytokines in an animal model of AD [84]. Recently, the Minocycline in Alzheimer's Disease Efficacy (MADE) trial compared two different doses of minocycline and placebo in mild $\mathrm{AD}$ patients over 2 years, but failed to demonstrate a beneficial effect [85]. The complexity of the relationship between microglial activation and neurodegeneration and the minimal treatment effects of minocycline were considered as reasons for the negative result [86]. CHF 5074 (CSP-1103) is a modulator of microglia that polarizes microglia from the neurotoxic to the neuroprotective phenotype [38]. A CHF 5074 trial assessing mild cognitive impairment following $\mathrm{AD}$ is currently in phase II (NCT01421056). Cromolyn, which is used in patients with asthma, has been found to induce neuroprotective microglial activation, promote $A \beta 42$ uptake in microglia, and reduce the aggregation-prone $A \beta$ levels [39]. The cromolyn is currently under a phase III trial (NCT02547818) as a therapeutic for early stages of $\mathrm{AD}$.

\section{PD}

$\mathrm{PD}$ is the most frequent movement disorder and the second most frequent neurodegenerative disease after $\mathrm{AD}$ [6]. The accumulation of Lewy bodies, intracellular inclusions that contain $\alpha$-synuclein, and dopaminergic neuronal death in the substantia nigra pars compacta and other brain regions are neuropathological hallmarks of PD $[6,87]$. In addition, the activation of glial cells, including microglia and astrocytes, also contributes to the pathogenesis of PD. Several proteins that are encoded by genes associated with familial forms of $\mathrm{PD}$, including $\alpha$ synuclein (PARK1 and PARK4), parkin (PARK2), DJ-1 (PARK7), and ATPase 13A2 (ATP13A2 gene), are involved in the regulation of microglial and astrocyte activation $[6,41]$.

The incremental activation of microglial cells (MHCII-, ICAM-1-, and LFA01-positive cells) is observed in the substantia nigra of PD patients [88]. Furthermore, the degree of microglial activation is correlated with the dopaminergic terminal loss in early PD [89]. The activated microglia that surround dopaminergic neurons are generally pro-inflammatory [28]. The aggregated $\alpha$ synuclein is released from dying dopaminergic neurons, and it activates microglia into the pro-inflammatory phenotype [90]. Over-expression of $\alpha$-synuclein drives microglia into a reactive pro-inflammatory phenotype, and TNF- $\alpha, N O$, and IL- $1 \beta$ derived from the proinflammatory microglia can modulate the neuroinflammatory process in PD [91, 92]. 1-Methyl-4-phenyl-1,2,3, 6-tetrahydropyridine is known to cause dopaminergic neuron injury via mitochondrial dysfunction and by indirectly activating microglia $[28,93]$. Lipopolysaccharide, a ligand of TLRs, can also cause dopaminergic neuronal death by activating the pro-inflammatory phenotype of microglia [28]. Jmjd3 has been reported to be essential for the expression of the M2 microglial phenotype [35]. Suppression of Jmjd3 attenuates the neuroprotective polarization and over-activates the pro-inflammatory microglial response with the exacerbation of dopaminergic neuronal cell death in a PD mouse model [35]. However, the role of the neuroprotective microglial phenotype is still unclear.

Reactive astrocytes have been detected in the substantia nigra pars compacta of PD patients [94]. Astrocyte 
dysfunction plays a role in dopaminergic neurodegeneration. Various genes are involved in the development of PD and astrocyte biology [95], including PARK7 (encoding DJ-1), SNCA (encoding $\alpha$-synuclein), PARK2 (encoding Parkin), PLA2G6 (encoding $\mathrm{Ca}^{2+}$-independent phospholipase $\mathrm{A}_{2}$ ), ATP13A2 (encoding lysosomal type 5 ATPase, ATP13A2), LRRK2 (encoding leucine-rich repeat kinase 2, LRRK2), GBA (encoding $\beta$-glucocerebrosidase, GCase), and PINK1 (encoding PTEN-induced putative kinase 1, PINK1) genes [95]. The DJ-1 protein regulates astrocyte activation through the IFN- $\gamma$ and TLR4 signaling $[41,96]$. Maintaining the ATP13A2 level could prevent the activation of the NLPR3 inflammasome [41, 95].

Various anti-inflammatory treatments such as dexamethasone, ibuprofen, amantadine, minocycline, pituitary adenylate cyclase-activating peptide, vasoactive intestinal peptide, IL-10, and TGF- $\beta$ have shown preventive effects on dopaminergic cell death in animal models [97-103]. However, the effects of antiinflammatory drugs in PD patients are contradictory. One meta-analysis concluded that the nonsteroidal antiinflammatory drugs (NSAIDs) may not modify the risk of PD, with only ibuprofen seeming to have a modest protective effect [104]. Another meta-analysis concluded that NSAIDs, except aspirin, may have a protective effect on the risk of PD [105]. Minocycline, which showed a neuroprotective effect in several in vivo and in vitro studies, was unsuccessful in altering the course of early PD over 12 and 18 months in a randomized clinical trial [106-108]. NLY01, a glucagon-like peptide-1 receptor agonist, was protective against dopaminergic neuronal loss and abnormal behavioral function in a sporadic PD mouse model [109]. This neuroprotective effect was attributed to the inhibition of the conversion of astrocytes to the neurotoxic phenotype, which was mediated by microglia [109].

\section{ALS}

ALS, also called Lou Gehrig's disease, is an adult-onset progressive neurodegenerative disease in which motor neurons are selectively affected [110]. The etiology of most ALS patients remains unidentified. Only less than $10 \%$ of cases are due to mutations of specific genes, including superoxidase dismutase 1 (SOD1), C9orf72, TDP43, and FUS [18]. Neuroinflammation is a pathological mechanism common to ALS patients with and without genetic mutations, which is characterized by the infiltration of activated microglia and astrocytes. The activated microglia and astrocytes that produce proinflammatory cytokines are upregulated in post-mortem tissues of ALS patients [41, 111, 112]. A PET study has demonstrated increases in activated microglia $\left[{ }^{11} \mathrm{C}\right.$ (R)PK11195 PET] and astrocytes $\left({ }^{11} \mathrm{C}\right.$-DED PET) in living ALS patients $[113,114]$. In addition, the CSF
sTREM2 level is significantly higher in sporadic ALS patients with varying disease severity than controls [115]. In particular, the CSF sTREM2 level is highest in the early-stage ALS, and in late stage, higher levels of CSF sTREM2 are associated with slower disease progression [115]. Prolonged high levels of CSF sTREM2 may be indicative of a neuroprotective phenotype.

Toxicity caused by mutant $S O D 1$, the most common form of inherited ALS, is mediated by direct damage that is incurred within the motor neurons, microglia, and astrocytes [110]. The activated pro-inflammatory microglia and astrocytes produce toxic factors that cause the initial damage and disease progression. The G930ASOD1 transgenic mouse model has demonstrated the ability of microglia to switch from the neuroprotective to the pro-inflammatory phenotype from the onset of the pathology $[28,116]$. The SOD1-mutant microglia isolated from mice with early-stage ALS express higher levels of M2 microglia phenotype markers and lower levels of pro-inflammatory microglia markers, compared with the SOD1-mutant microglia isolated from mice with end-stage ALS [117]. Altogether, as ALS progresses, the function of neuroprotective microglia may decrease and the proportion of pro-inflammatory phenotypes may increase. The C3-expressing pro-inflammatory astrocytes and astrocytic NLRP3 inflammasomes have been found to be upregulated in post-mortem ALS patients [41, 46, 118]. The activation of astrocytes in ALS decreases their protective effects and increases their detrimental effects [119]. Astrocytes with SOD1 mutations have been reported to release soluble factors toxic to motor neurons [120]. IL-1 $\alpha$, TNF- $\alpha$, and C1q released from microglia drive astrocytes to the neurotoxic phenotype, while reducing reactive astrocytes by inhibiting these factors attenuates the disease progression in the G93A-SOD1 mouse model [121]. However, little is known about the neuroprotective phenotype of astrocytes in the pathogenesis of ALS.

The ablation of NOX3 or NF-kB improved motor neuron survival in the G930A-SOD1 transgenic mouse model $[28,122]$. In addition, the administration of minocycline in G930A-SOD1 transgenic mice selectively attenuated the expression of markers for the proinflammatory microglia, inhibited the upregulation of $\mathrm{NF}-\mathrm{\kappa B}$ in the primary culture of microglia, and delayed the pathogenesis $[28,123]$. Cromolyn, which induced the activation of neuroprotective microglia in the $\mathrm{AD}$ mouse model, demonstrated a neuroprotective effect in the G93A-SOD1 transgenic mouse model by delaying the disease onset and reducing the motor impairment [124].

Recently, masitinib, an oral tyrosine kinase inhibitor, has shown beneficial effects in ALS patients over 48 weeks [125]. Masitinib reduces the microgliosis and the 
emergence of aberrant glial cells in the G93A-SOD1 transgenic mouse model [126]. Regulatory Tlymphocytes (Tregs) can augment IL-4 expression, induce the M2-phenotype microglia, and delay the progression of the disease [127]. Upregulation of Tregs can be achieved using dimethyl fumarate (Tecfidera), and a phase II trial of Tecfidera is being conducted in patients with sporadic ALS [128]. In addition, the infusion of tocilizumab in ALS patients reduced neuroinflammation [129], and it is now in a phase II clinical trial in ALS patients (NCT02469896), results of which are expected to be announced soon.

\section{Conclusion}

We reviewed the roles of neuroinflammation in neurodegenerative diseases, focusing on microglia and astrocytes. In addition, clinical or experimental studies on treatments associated with neuroinflammation in neurodegenerative diseases were discussed. A balance between pro-inflammatory and neuroprotective glial cells may be critical in the progression of neurodegenerative diseases. Moreover, it has been reported that the activated microglia and reactive astrocytes influence each other. Due to the complexity of microglia and astrocyte phenotypes and the various types of drugs, the stages of neurodegenerative diseases (more pro-inflammatory than neuroprotective) and the conditions of patients (confirmed pathology of disease and likely to progress within few years) may be crucial for demonstrating the benefits of anti-inflammatory treatments in clinical trials. The functions of microglia and astrocytes at specific stages of specific diseases in specific patients need to be identified. The next step for trials is to determine a standard method for evaluating each phenotype of microglia and astrocytes to standardize further evaluation.

\footnotetext{
Abbreviations

${ }^{11}$ C-DED: ${ }^{11}$ C-deuterium-L-deprenyl; A 3 : Amyloid-beta; Act 1: Activator 1; AD: Alzheimer's disease; ALS: Amyotrophic lateral sclerosis; Arg1: Arginase 1; BBB: Blood-brain barrier; BDNF: Brain-derived neurotrophic factor; Chi3l3: Chitinase-3-Like-3; CCL: C-C motif chemokine ligand; CSF: Cerebrospinal fluid; CXCL: C-X-C motif chemokine ligand; DAMPs: damage-associated molecular patterns; EAE: Experimental autoimmune encephalomyelitis; ER: Oestrogen receptor; Fzd1: Frizzled class receptor 1; GFAP: Glial fibrillary acidic protein; GM-CSF: Granulocytemacrophage colony-stimulating factor; IFN: Interferon; IGF-1: Insulin-like growth factor 1; IL: Interleukin; JAK/STAT: Janus kinase/signal transducers and activators of transcription; Jmjd3: Jumonji domain containing 3; LacCer: Lactosylceraide; LPS: Lipopolysaccharide; NFkB: Nuclear factor kB; NO: Nitric oxide; PAMP: Pathogen-associated molecular pattern; PD: Parkinson's disease; PET: Positron emission tomography; SCI: Spinal cord injury; SOD1: Superoxidase dismutase 1; STAT3: Signal transducer and activator of transcription 3; sTREM2: Soluble triggering receptor expressed on myeloid cells 2; TGF: Transforming growth factor; TLR: Toll-like receptor; TNF: Tumor necrosis factor; TrkB: Tropomyosin receptor kinase B; Tregs: Regulatory $\mathrm{T}$ cells
}

\section{Acknowledgments}

We would like to thank Editage (www.editage.co.kr) for English language editing.

\section{Authors' contributions}

KSH conceived the idea of this review. KHS drafted the manuscript and created the figures. KHS and KSH performed the literature search and reviewed the content of this manuscript. The authors read and approved the final manuscript.

\section{Author's information}

Not applicable.

\section{Funding}

This review was supported by the Basic Science Research Program of the National Research Foundation of Korea, which was funded by the Ministry of Science, ICT, and Future Planning (2018R1A2A2A15023219), a grant of the Korea Health Technology R\&D Project through the Korea Health Industry Development Institute (KHIDI) funded by the Ministry of Health \& Welfare, Republic of Korea (HI20C0253), and the Medical Research Centre (2017R1A5A2015395).

\section{Availability of data and materials}

Not applicable.

Ethics approval and consent to participate

Not applicable.

\section{Consent for publication}

Not applicable.

\section{Competing interests}

The authors declare that they have no competing interests.

Received: 16 June 2020 Accepted: 3 November 2020 Published online: 26 November 2020

\section{References}

1. Cova I, Markova A, Campini I, Grande G, Mariani C, Pomati S. Worldwide trends in the prevalence of dementia. J Neurol Sci. 2017;379:259-60.

2. Jellinger KA. Basic mechanisms of neurodegeneration: a critical update. J Cell Mol Med. 2010;14:457-87.

3. Wyss-Coray T, Mucke L. Inflammation in neurodegenerative disease--a double-edged sword. Neuron. 2002;35:419-32.

4. Kempuraj D, Thangavel R, Natteru PA, Selvakumar GP, Saeed D, Zahoor H, et al. Neuroinflammation induces neurodegeneration. J Neurol Neurosurg Spine. 2016;1:1003.

5. Russo MV, McGavern DB. Inflammatory neuroprotection following traumatic brain injury. Science. 2016;353:783-5.

6. Glass CK, Saijo K, Winner B, Marchetto MC, Gage FH. Mechanisms underlying inflammation in neurodegeneration. Cell. 2010;140:918-34.

7. Stephenson J, Nutma E, van der Valk P, Amor S. Inflammation in CNS neurodegenerative diseases. Immunology. 2018;154:204-19.

8. Subhramanyam CS, Wang C, Hu Q, Dheen ST. Microglia-mediated neuroinflammation in neurodegenerative diseases. Semin Cell Dev Biol. 2019:94:112-20.

9. Fields RD, Araque A, Johansen-Berg H, Lim SS, Lynch G, Nave KA, et al. Glial biology in learning and cognition. Neuroscientist. 2014;20:426-31.

10. Clarke LE, Barres BA. Emerging roles of astrocytes in neural circuit development. Nat Rev Neurosci. 2013;14:311-21.

11. Luo XG, Chen SD. The changing phenotype of microglia from homeostasis to disease. Transl Neurodegener. 2012;1:9.

12. Bachiller S, Jiménez-Ferrer I, Paulus A, Yang Y, Swanberg M, Deierborg T, et al. A microglia in neurological diseases: a road map to brain-disease dependent-inflammatory response. Front Cell Neurosci. 2018;12:488.

13. Liddelow SA, Barres BA. Reactive astrocytes: production, function, and therapeutic potential. Immunity. 2017;46:957-67.

14. De Biase LM, Schuebel KE, Fusfeld ZH, Jair K, Hawes IA, Cimbro R, et al. Local cues establish and maintain region-specific phenotypes of basal ganglia microglia. Neuron. 2017;95:341-56.

15. Heneka MT, Carson MJ, El Khoury J, Landreth GE, Brosseron F, Feinstein DL, et al. Neuroinflammation in Alzheimer's disease. Lancet Neurol. 2015;14:388-405.

16. Baufeld C, O'Loughlin E, Calcagno N, Madore C, Butovsky O. Differential contribution of microglia and monocytes in neurodegenerative diseases. Neural Transm. 2018;125:809-26. 
17. Lawson LJ, Perry VH, Dri P, Gordon S. Heterogeneity in the distribution and morphology of microglia in the normal adult mouse brain. Neuroscience. 1990;39:151-70

18. Hickman S, Izzy S, Sen P, Morsett L, El Khoury J. Microglia in neurodegeneration. Nat Neurosci. 2018;21:1359-69.

19. Hickman SE, Kingery ND, Ohsumi TK, Borowsky ML, Wang LC, Means TK, et al. The microglial sensome revealed by direct RNA sequencing. Nat Neurosci. 2013;16:1896-905.

20. Zhan Y, Paolicelli RC, Sforazzini F, Weinhard L, Bolasco G, Pagani F, et al. Deficient neuron-microglia signaling results in impaired functional brain connectivity and social behavior. Nat Neurosci. 2014;17:400-6.

21. Niraula A, Sheridan JF, Godbout JP. Microglia priming with aging and stress. Neuropsychopharmacology. 2017;42:318-33

22. Scarf AM, Kassiou M. The translocator protein. J Nucl Med. 2011;52:677-80.

23. Malpetti M, Kievit RA, Passamonti L, Jones PS, Tsvetanov KA, Rittman T, et al. Microglial activation and tau burden predict cognitive decline in Alzheimer's disease. Brain. 2020;143:1588-602.

24. Kwon HS, Lee EH, Park HH, Jin JH, Choi H, Lee KY, et al. Early increment of soluble triggering receptor expressed on myeloid cells 2 in plasma might be a predictor of poor outcome after ischemic stroke. J Clin Neurosci. 2020; 73:215-8.

25. Bekris LM, Khrestian M, Dyne E, Shao Y, Pillai JA, Rao SM, et al. Soluble TREM2 and biomarkers of central and peripheral inflammation in neurodegenerative disease. J Neuroimmunol. 2018;319:19-27.

26. Suárez-Calvet M, Kleinberger $G$, Araque Caballero M, Brendel M, Rominger A, Alcolea D, et al. sTREM2 cerebrospinal fluid levels are a potential biomarker for microglia activity in early-stage Alzheimer's disease and associate with neuronal injury markers. EMBO Mol Med. 2016;8:466-76.

27. Sica A, Mantovani A. Macrophage plasticity and polarization: in vivo veritas. J Clin Invest. 2012;122:787-95.

28. Tang $Y$, Le $W$. Differential roles of $M 1$ and $M 2$ microglia in neurodegenerative diseases. Mol Neurobiol. 2016;53:1181-94

29. Zhao W, Xie W, Xiao Q, Beers DR, Appel SH. Protective effects of an antiinflammatory cytokine, interleukin-4, on motoneuron toxicity induced by activated microglia. J Neurochem. 2006;99:1176-87.

30. Park KW, Lee DY, Joe EH, Kim SU, Jin BK. Neuroprotective role of microglia expressing interleukin-4. J Neurosci Res. 2005;81:397-402.

31. Ruckh JM, Zhao JW, Shadrach JL, van Wijngaarden P, Rao TN, Wagers AJ, et al. Rejuvenation of regeneration in the aging central nervous system. Cell Stem Cell. 2012;10:96-103.

32. Lumeng CN, Bodzin JL, Saltiel AR. Obesity induces a phenotypic switch in adipose tissue macrophage polarization. J Clin Invest. 2007;117:175-84.

33. Odegaard Jl, Ricardo-Gonzalez RR, Goforth MH, Morel CR, Subramanian V, Mukundan L, et al. Macrophage-specific PPARgamma controls alternative activation and improves insulin resistance. Nature. 2007:447:1116-20.

34. Zhang H, Li Y, Yu J, Guo M, Meng J, Liu C, et al. Rho kinase inhibitor fasudil regulates microglia polarization and function. Neuroimmunomodulation. 2013;20:313-22

35. Tang Y, Li T, Li J, Yang J, Liu H, Zhang XJ, et al. Jmjd3 is essential for the epigenetic modulation of microglia phenotypes in the immune pathogenesis of Parkinson's disease. Cell Death Differ. 2014;21:369-80.

36. Kim SH, Noh MY, Kim HJ, Oh KW, Park J, Lee S, et al. A therapeutic strategy for Alzheimer's disease focused on immune-inflammatory modulation. Dement Neurocogn Disord. 2019;18:33-46.

37. Miao H, Li R, Han C, Lu X, Zhang H. Minocycline promotes posthemorrhagic neurogenesis via M2 microglia polarization via upregulation of the TrkB/ BDNF pathway in rats. J Neurophysiol. 2018;120:1307-17.

38. Porrini V, Lanzillotta A, Branca C, Benarese M, Parrella E, Lorenzini L, et al. CHF5074 (CSP-1103) induces microglia alternative activation in plaque-free Tg2576 mice and primary glial cultures exposed to beta-amyloid. Neuroscience. 2015;302:112-20.

39. Zhang C, Griciuc A, Hudry E, Wan Y, Quinti L, Ward J, et al. Cromolyn reduces levels of the Alzheimer's disease-associated amyloid beta-protein by promoting microglial phagocytosis. Sci Rep. 2018;8:1144.

40. Colombo E, Farina C. Astrocytes: key regulators of neuroinflammation. Trends Immunol. 2016;37:608-20.

41. Oksanen M, Lehtonen S, Jaronen M, Goldsteins G, Hamalainen RH Koistinaho J. Astrocyte alterations in neurodegenerative pathologies and their modeling in human induced pluripotent stem cell platforms. Cell Mo Life Sci. 2019;76:2739-60.
42. Sofroniew MV. Molecular dissection of reactive astrogliosis and glial scar formation. Trends Neurosci. 2009;32:638-47.

43. Carter SF, Herholz K, Rosa-Neto P, Pellerin L, Nordberg A, Zimmer ER. Astrocyte biomarkers in Alzheimer's disease. Trends Mol Med. 2019;25:77-95.

44. Mayo L, Trauger SA, Blain M, Nadeau M, Patel B, Alvarez Jl, et al. Regulation of astrocyte activation by glycolipids drives chronic CNS inflammation. Nat Med. 2014;20:1147-56

45. Saijo K, Winner B, Carson CT, Collier JG, Boyer L, Rosenfeld MG, et al. A Nurr1/CoREST pathway in microglia and astrocytes protects dopaminergic neurons from inflammation-induced death. Cell. 2009;137:47-59.

46. Liddelow SA, Guttenplan KA, Clarke LE, Bennett FC, Bohlen CJ, Schirmer L, et al. Neurotoxic reactive astrocytes are induced by activated microglia. Nature. 2017:541:481-7.

47. Qian Y, Liu C, Hartupee J, Altuntas CZ, Gulen MF, Jane-Wit D, et al. The adaptor Act1 is required for interleukin 17-dependent signaling associated with autoimmune and inflammatory disease. Nat Immunol. 2007:8:247-56.

48. Colombo E, Cordiglieri C, Melli G, Newcombe J, Krumbholz M, Parada LF, et al. Stimulation of the neurotrophin receptor TrkB on astrocytes drives nitric oxide production and neurodegeneration. J Exp Med. 2012;209:521-35.

49. Haroon F, Drogemuller K, Handel U, Brunn A, Reinhold D, Nishanth G, et al. Gp130-dependent astrocytic survival is critical for the control of autoimmune central nervous system inflammation. J Immunol. 2011;186: 6521-31.

50. Drogemuller K, Helmuth U, Brunn A, Sakowicz-Burkiewicz M, Gutmann DH, Mueller W, et al. Astrocyte gp130 expression is critical for the control of toxoplasma encephalitis. J Immunol. 2008;181:2683-93.

51. Cekanaviciute E, Fathali N, Doyle KP, Williams AM, Han J, Buckwalter MS Astrocytic transforming growth factor-beta signaling reduces subacute neuroinflammation after stroke in mice. Glia. 2014;62:1227-40.

52. Cekanaviciute E, Dietrich HK, Axtell RC, Williams AM, Egusquiza R, Wai KM, et al. Astrocytic TGF-beta signaling limits inflammation and reduces neuronal damage during central nervous system toxoplasma infection. J Immunol. 2014;193:139-49.

53. Hindinger C, Bergmann CC, Hinton DR, Phares TW, Parra Gl, Hussain S, et al. IFN-gamma signaling to astrocytes protects from autoimmune mediated neurological disability. PLoS One. 2012;7:e42088.

54. Tiwari-Woodruff S, Morales LB, Lee R, Voskuhl RR. Differential neuroprotective and antiinflammatory effects of estrogen receptor (ER) alpha and ERbeta ligand treatment. Proc Natl Acad Sci U S A. 2007;104: 14813-8.

55. Okada S, Nakamura M, Katoh H, Miyao T, Shimazaki T, Ishii K, et al. Conditional ablation of Stat3 or Socs3 discloses a dual role for reactive astrocytes after spinal cord injury. Nat Med. 2006;12:829-34.

56. Islam O, Loo TX, Heese K. Brain-derived neurotrophic factor (BDNF) has proliferative effects on neural stem cells through the truncated TRK-B receptor, MAP kinase, AKT, and STAT-3 signaling pathways. Curr NeurovasC Res. 2009:6:42-53.

57. Herrmann JE, Imura T, Song B, Qi J, Ao Y, Nguyen TK, et al. STAT3 is a critical regulator of astrogliosis and scar formation after spinal cord injury. J Neurosci. 2008;28:7231-43.

58. Ceyzeriat K, Abjean L, Carrillo-de Sauvage MA, Ben Haim L, Escartin C. The complex STATes of astrocyte reactivity: how are they controlled by the JAKSTAT3 pathway? Neuroscience. 2016;330:205-18.

59. Prince M, Ali GC, Guerchet M, Prina AM, Albanese E, Wu YT. Recent global trends in the prevalence and incidence of dementia, and survival with dementia. Alzheimers Res Ther. 2016;8:23.

60. Tarkowski E, Andreasen N, Tarkowski A, Blennow K. Intrathecal inflammation precedes development of Alzheimer's disease. J Neurol Neurosurg Psychiatry. 2003;74:1200-5.

61. Brosseron F, Krauthausen M, Kummer M, Heneka MT. Body fluid cytokine levels in mild cognitive impairment and Alzheimer's disease: a comparative overview. Mol Neurobiol. 2014;50:534-44.

62. Yan $\sqcup$, Xiao M, Chen R, Cai Z. Metabolic dysfunction of astrocyte: an initiating factor in beta-amyloid pathology? Aging Neurodegener. 2013;1:7-14.

63. Takata K, Kitamura Y, Saeki M, Terada M, Kagitani S, Kitamura R, et al. Galantamine-induced amyloid-\{beta\} clearance mediated via stimulation of microglial nicotinic acetylcholine receptors. J Biol Chem. 2010;285: 40180-91.

64. Asai H, Ikezu S, Tsunoda S, Medalla M, Luebke J, Haydar T, et al. Depletion of microglia and inhibition of exosome synthesis halt tau propagation. Nat Neurosci. 2015;18:1584-93. 
65. Mawuenyega KG, Sigurdson W, Ovod V, Munsell L, Kasten T, Morris JC, et al. Decreased clearance of CNS beta-amyloid in Alzheimer's disease. Science. 2010;330:1774

66. Jimenez S, Baglietto-Vargas D, Caballero C, Moreno-Gonzalez I, Torres M, Sanchez-Varo R, et al. Inflammatory response in the hippocampus of PS1M146L/APP751SL mouse model of Alzheimer's disease: age-dependent switch in the microglial phenotype from alternative to classic. J Neurosci. 2008;28:11650-61.

67. Lee DC, Rizer J, Selenica ML, Reid P, Kraft C, Johnson A, et al. LPS- induced inflammation exacerbates phospho-tau pathology in rTg4510 mice. J Neuroinflammation. 2010;7:56.

68. Fan Z, Brooks DJ, Okello A, Edison P. An early and late peak in microglial activation in Alzheimer's disease trajectory. Brain. 2017;140:792-803.

69. Varnum MM, Ikezu T. The classification of microglial activation phenotypes on neurodegeneration and regeneration in Alzheimer's disease brain. Arch Immunol Ther Exp (Warsz). 2012;60:251-66.

70. Carmona S, Zahs K, Wu E, Dakin K, Bras J, Guerreiro R. The role of TREM2 in Alzheimer's disease and other neurodegenerative disorders. Lancet Neurol. 2018;17:721-30.

71. Rothstein JD, Dykes-Hoberg M, Pardo CA, Bristol LA, Jin L, Kund RW, et al. Knockout of glutamate transporters reveals a major role for astroglial transport in excitotoxicity and clearance of glutamate. Neuron. 1996;16:675-86.

72. Sompol P, Furman JL, Pleiss MM, Kraner SD, Artiushin IA, Batten SR, et al. Calcineurin/NFAT signaling in activated astrocytes drives network hyperexcitability in Abeta-bearing mice. J Neurosci. 2017;37:6132-48.

73. Kellner V, Menkes-Caspi N, Beker S, Stern EA. Amyloid-beta alters ongoing neuronal activity and excitability in the frontal cortex. Neurobiol Aging. 2014:35:1982-91.

74. Aisen PS, Davis KL, Berg JD, Schafer K, Campbell K, Thomas RG, et al. A randomized controlled trial of prednisone in Alzheimer's disease. Alzheimer's disease cooperative study. Neurology. 2000;54:588-93.

75. Aisen PS, Schafer KA, Grundman M, Pfeiffer E, Sano M, Davis KL, et al. Effects of rofecoxib or naproxen vs placebo on Alzheimer disease progression: a randomized controlled trial. JAMA. 2003;289:2819-26.

76. Bentham P, Gray R, Sellwood E, Hills R, Crome P, Raftery J. Aspirin in Alzheimer's disease (AD2000): a randomised open-label trial. Lancet Neurol. 2008;7:41-9.

77. Scharf S, Mander A, Ugoni A, Vajda F, Christophidis N. A double-blind, placebo-controlled trial of diclofenac/misoprostol in Alzheimer's disease. Neurology. 1999:53:197-201.

78. Thal $\amalg$, Ferris SH, Kirby L, Block GA, Lines CR, Yuen E, et al. A randomized, double-blind, study of rofecoxib in patients with mild cognitive impairment. Neuropsychopharmacology. 2005;30:1204-15.

79. Martin BK, Szekely C, Brandt J, Piantadosi S, Breitner JC, Craft S, et al. Cognitive function over time in the Alzheimer's disease anti-inflammatory prevention trial (ADAPT): results of a randomized, controlled trial of naproxen and celecoxib. Arch Neurol. 2008;65:896-905.

80. Pasqualetti P, Bonomini C, Dal Forno G, Paulon L, Sinforiani E, Marra C, et al. A randomized controlled study on effects of ibuprofen on cognitive progression of Alzheimer's disease. Aging Clin Exp Res. 2009;21:102-10.

81. Rogers J, Kirby LC, Hempelman SR, Berry DL, McGeer PL, Kaszniak AW, et al. Clinical trial of indomethacin in Alzheimer's disease. Neurology. 1993;43: 1609-11.

82. Krause DL, Müller N. Neuroinflammation, microglia and implications for antiinflammatory treatment in Alzheimer's disease. Int J Alzheimers Dis. 2010; 2010:732806.

83. Familian A, Boshuizen RS, Eikelenboom P, Veerhuis R. Inhibitory effect of minocycline on amyloid beta fibril formation and human microglial activation. Glia. 2006;53:233-40.

84. Garcez ML, Mina F, Bellettini-Santos T, Carneiro FG, Luz AP, Schiavo GL, et al. Minocycline reduces inflammatory parameters in the brain structures and serum and reverses memory impairment caused by the administration of amyloid beta (1-42) in mice. Prog Neuropsychopharmacol Biol Psychiatry. 2017;77:23-31.

85. Howard R, Zubko O, Bradley R, Harper E, Pank L, O'Brien J, et al. Minocycline at 2 different dosages vs placebo for patients with mild Alzheimer disease: a randomized clinical trial. JAMA Neurol. 2019;77:164-74.

86. Gyengesi E, Munch $\mathrm{G}$. In search of an anti-inflammatory drug for Alzheimer disease. Nat Rev Neurol. 2020;16:131-2.

87. Braak H, Del Tredici K, Rub U, de Vos RA, Jansen Steur EN, Braak E. Staging of brain pathology related to sporadic Parkinson's disease. Neurobiol Aging. 2003;24:197-211.
88. Rocha NP, de Miranda AS, Teixeira AL. Insights into neuroinflammation in Parkinson's disease: from biomarkers to anti-inflammatory based therapies. Biomed Res Int. 2015;2015:628192.

89. Ouchi Y, Yoshikawa E, Sekine Y, Futatsubashi M, Kanno T, Ogusu T, et al. Microglial activation and dopamine terminal loss in early Parkinson's disease. Ann Neurol. 2005;57:168-75.

90. Zhang W, Wang T, Pei Z, Miller DS, Wu X, Block ML, et al. Aggregated alphasynuclein activates microglia: a process leading to disease progression in Parkinson's disease. FASEB J. 2005:19:533-42.

91. Rojanathammanee L, Murphy EJ, Combs CK. Expression of mutant alphasynuclein modulates microglial phenotype in vitro. J Neuroinflammation. 2011:8:44.

92. Hirsch EC, Hunot S. Neuroinflammation in Parkinson's disease: a target for neuroprotection? Lancet Neurol. 2009;8:382-97.

93. Dauer W, Przedborski S. Parkinson's disease: mechanisms and models. Neuron. 2003;39:889-909.

94. Miklossy J, Doudet DD, Schwab C, Yu S, McGeer EG, McGeer PL. Role of ICAM-1 in persisting inflammation in Parkinson disease and MPTP monkeys. Exp Neurol. 2006;197:275-83.

95. Booth HDE, Hirst WD, Wade-Martins R. The role of astrocyte dysfunction in Parkinson's disease pathogenesis. Trends Neurosci. 2017;40:358-70.

96. Kim JH, Choi DJ, Jeong HK, Kim J, Kim DW, Choi SY, et al. DJ-1 facilitates the interaction between STAT1 and its phosphatase, SHP-1, in brain microglia and astrocytes: a novel anti-inflammatory function of DJ-1. Neurobiol Dis. 2013;60:1-10

97. Manthripragada AD, Schernhammer ES, Qiu J, Friis S, Wermuth L, Olsen JH, et al. Non-steroidal anti-inflammatory drug use and the risk of Parkinson's disease. Neuroepidemiology. 2011;36:155-61.

98. Gao X, Chen H, Schwarzschild MA, Ascherio A. Use of ibuprofen and risk of Parkinson disease. Neurology. 2011;76:863-9.

99. Malagelada C, Jin ZH, Jackson-Lewis V, Przedborski S, Greene LA. Rapamycin protects against neuron death in in vitro and in vivo models of Parkinson's disease. J Neurosci. 2010;30:1166-75.

100. Delgado M, Jonakait GM, Ganea D. Vasoactive intestinal peptide and pituitary adenylate cyclase-activating polypeptide inhibit chemokine production in activated microglia. Glia. 2002;39:148-61.

101. Lamine A, Letourneau M, Doan ND, Maucotel J, Couvineau A, Vaudry H, et al. Characterizations of a synthetic pituitary adenylate cyclase-activating polypeptide analog displaying potent neuroprotective activity and reduced in vivo cardiovascular side effects in a Parkinson's disease model. Neuropharmacology. 2016;108:440-50.

102. Qian L, Block ML, Wei SJ, Lin CF, Reece J, Pang H, et al. Interleukin-10 protects lipopolysaccharide-induced neurotoxicity in primary midbrain cultures by inhibiting the function of NADPH oxidase. J Pharmacol Exp Ther. 2006:319:44-52.

103. Zhu Y, Yang GY, Ahlemeyer B, Pang L, Che XM, Culmsee C, et al. Transforming growth factor-beta 1 increases bad phosphorylation and protects neurons against damage. J Neurosci. 2002;22:3898-909.

104. Samii A, Etminan M, Wiens MO, Jafari S. NSAID use and the risk of Parkinson's disease: systematic review and meta-analysis of observational studies. Drugs Aging. 2009:26:769-79.

105. Gagne JJ, Power MC. Anti-inflammatory drugs and risk of Parkinson disease: a meta-analysis. Neurology. 2010;74:995-1002

106. NINDS NET-PD Investigators. A randomized, double-blind, futility clinical trial of creatine and minocycline in early Parkinson disease. Neurology. 2006;66:664-71.

107. NINDS NET-PD Investigators. A pilot clinical trial of creatine and minocycline in early Parkinson disease: 18-month results. Clin Neuropharmacol. 2008;31: $141-50$

108. Cankaya S, Cankaya B, Kilic U, Kilic E, Yulug B. The therapeutic role of minocycline in Parkinson's disease. Drugs Context. 2019;8:212553.

109. Yun SP, Kam TI, Panicker N, Kim S, Oh Y, Park JS, et al. Block of A1 astrocyte conversion by microglia is neuroprotective in models of Parkinson's disease. Nat Med. 2018;24:931-8

110. Boillee S, Vande Velde C, Cleveland DW. ALS: a disease of motor neurons and their nonneuronal neighbors. Neuron. 2006;52:39-59.

111. Henkel JS, Engelhardt JI, Siklos L, Simpson EP, Kim SH, Pan T, et al. Presence of dendritic cells, MCP-1, and activated microglia/macrophages in amyotrophic lateral sclerosis spinal cord tissue. Ann Neurol. 2004:55:221-35.

112. Kawamata T, Akiyama H, Yamada T, McGeer PL. Immunologic reactions in amyotrophic lateral sclerosis brain and spinal cord tissue. Am J Pathol. 1992; 140:691-707. 
113. Turner MR, Cagnin A, Turkheimer FE, Miller CC, Shaw CE, Brooks DJ, et al. Evidence of widespread cerebral microglial activation in amyotrophic lateral sclerosis: an [11C](R)-PK11195 positron emission tomography study. Neurobiol Dis. 2004:15:601-9.

114. Johansson A, Engler H, Blomquist G, Scott B, Wall A, Aquilonius SM, et al. Evidence for astrocytosis in ALS demonstrated by [11C](L)-deprenyl-D2 PET. J Neurol Sci. 2007;255:17-22.

115. Cooper-Knock J, Green C, Altschuler G, Wei W, Bury JJ, Heath PR, et al. A data-driven approach links microglia to pathology and prognosis in amyotrophic lateral sclerosis. Acta Neuropathol Commun. 2017;5:23.

116. Hensley K, Fedynyshyn J, Ferrell S, Floyd RA, Gordon B, Grammas P, et al. Message and protein-level elevation of tumor necrosis factor alpha (TNF alpha) and TNF alpha-modulating cytokines in spinal cords of the G93ASOD1 mouse model for amyotrophic lateral sclerosis. Neurobiol Dis. 2003; 14:74-80.

117. Liao B, Zhao W, Beers DR, Henkel JS, Appel SH. Transformation from a neuroprotective to a neurotoxic microglial phenotype in a mouse model of ALS. Exp Neurol. 2012;237:147-52.

118. Johann S, Heitzer M, Kanagaratnam M, Goswami A, Rizo T, Weis J, et al. NLRP3 inflammasome is expressed by astrocytes in the SOD1 mouse model of ALS and in human sporadic ALS patients. Glia. 2015;63:2260-73.

119. Yamanaka K, Komine O. The multi-dimensional roles of astrocytes in ALS. Neurosci Res. 2018;126:31-8.

120. Nagai M, Re DB, Nagata T, Chalazonitis A, Jessell TM, Wichterle $H$, et al. Astrocytes expressing ALS-linked mutated SOD1 release factors selectively toxic to motor neurons. Nat Neurosci. 2007:10:615-22.

121. Guttenplan KA, Weigel MK, Adler DI, Couthouis J, Liddelow SA, Gitler AD, et al. Knockout of reactive astrocyte activating factors slows disease progression in an ALS mouse model. Nat Commun. 2020;1 1:3753.

122. Frakes AE, Ferraiuolo L, Haidet-Phillips AM, Schmelzer L, Braun L, Miranda CJ, et al. Microglia induce motor neuron death via the classical NF-kappaB pathway in amyotrophic lateral sclerosis. Neuron. 2014;81:1009-23.

123. Kobayashi K, Imagama S, Ohgomori T, Hirano K, Uchimura K, Sakamoto K, et al. Minocycline selectively inhibits M1 polarization of microglia. Cell Death Dis. 2013:4:e525.

124. Granucci EJ, Griciuc A, Mueller KA, Mills AN, Le H, Dios AM, et al. Cromolyn sodium delays disease onset and is neuroprotective in the SOD1(G93A) mouse model of amyotrophic lateral sclerosis. Sci Rep. 2019;9:17728.

125. Mora JS, Genge A, Chio A, Estol CJ, Chaverri D, Hernandez M, et al. Masitinib as an add-on therapy to riluzole in patients with amyotrophic lateral sclerosis: a randomized clinical trial. Amyotroph Lateral Scler Frontotemporal Degener. 2020;21:5-14

126. Trias E, Ibarburu S, Barreto-Nunez R, Babdor J, Maciel TT, Guillo M, et al. Post-paralysis tyrosine kinase inhibition with masitinib abrogates neuroinflammation and slows disease progression in inherited amyotrophic lateral sclerosis. J Neuroinflammation. 2016;13:177.

127. Beers DR, Henkel JS, Zhao W, Wang J, Huang A, Wen S, et al. Endogenous regulatory $\mathrm{T}$ lymphocytes ameliorate amyotrophic lateral sclerosis in mice and correlate with disease progression in patients with amyotrophic lateral sclerosis. Brain. 2011:134:1293-314.

128. Vucic S, Ryder J, Mekhael L, Rd H, Mathers S, Needham M, et al. Phase 2 randomized placebo controlled double blind study to assess the efficacy and safety of tecfidera in patients with amyotrophic lateral sclerosis (TEALS study): study protocol clinical trial (SPIRIT compliant). Medicine. 2020;99: e18904.

129. Mizwicki MT, Fiala M, Magpantay L, Aziz N, Sayre J, Liu G, et al. Tocilizumab attenuates inflammation in ALS patients through inhibition of IL6 receptor signaling. Am J Neurodegener Dis. 2012;1:305-15.

\section{Ready to submit your research? Choose BMC and benefit from:}

- fast, convenient online submission

- thorough peer review by experienced researchers in your field

- rapid publication on acceptance

- support for research data, including large and complex data types

- gold Open Access which fosters wider collaboration and increased citations

- maximum visibility for your research: over $100 \mathrm{M}$ website views per year

At BMC, research is always in progress.

Learn more biomedcentral.com/submissions 